\title{
A Spatiotemporal data acquisition and processing method for Ansai waist drum based on motion capture
}

\author{
YingGuo $^{1}$, Xupeng Wang ${ }^{1 *}$ \\ ${ }^{1}$ Department of Industrial Design, Xi'an University of Technology, Xi'an Shaanxi, 710048, China
}

\begin{abstract}
In order to effectively promote the inheritance and preservation of the intangible cultural heritage project-Ansai waist drum. Based on motion capture systems, a spatiotemporal data acquisition and processing method is introduced in this works, which contains principles and specific methods for data acquisition and processing. Take a typical action as an example, the method is presented seriously, which refers to acquisition site, motion capture equipment, real-time acquisition, data output, post-processing software, as well as skeleton and excel data processing. Furthermore, the canonical motion data of Ansai waist drum also can be calculated by this method, and then used as the standard data for beginners. The method could be a reference for inheritance and preservation to sports and dance intangible cultural heritage projects.
\end{abstract}

\section{Introduction}

The Ansai waist drum is known as "the first drum in China", which integrates dance, gymnastics, percussion, and playing music, as well as martial arts and folk songs. It was listed in the first batch of national intangible cultural heritage on May 20, 2006 [1]. Although Ansai waist drum is well-known at home and abroad, there are many problems in its inheritance and protection. Especially, the inheritance mode is single and mainly contains the master-apprentice style and family style [2]. And the ancient traditions are in danger of losing. Due to the emphasis on commercial development, the role of Ansai waist drum changed from sanctification of blessing ceremony to secular utilitarianism. And the military waist drum, which represents the symbol of war, gradually lost its inheritance [3]. These problems have seriously hindered the inheritance and protection of Ansai waist drum, which need to be solved urgently.

A larger number of researchers focus on the inheritance and protection of national intangible cultural heritage based on digital way. Based on the threedimensional virtual interactive platform, Marcello Carrozzino et al. realized the digital display and demonstration teaching of traditional crafts such as handicrafts and sculpture [4]. Jacky C.R Chan et al. developed a dancing study system using motion capture and virtual reality technology, which can capture and analyze dance movements of beginners, then provide feedback and realize interactive learning [5]. Lei Shi studied the interactive display and digital protection of Huayaoyi dance with the help of motion capture technology and three-dimensional animation [6]. Tao Yu input the standard movement data and set up several standard movement data sets of 24 styles Taijiquan based on motion capture [7]. Junyi Chen used virtual humancomputer interaction technology and designed a multi platform interactive game of Guanglin paper-cut, which realizes the dissemination and protection of Guangling paper-cut art [8].

The paper focuses on introducing a method for acquisition and processing Spatiotemporal data of Ansai waist drum based on motion capture technology, which can provide help for the digital and standardized inheritance of Ansai waist drum. The second part of the paper introduces the test scheme of spatiotemporal data in inheritance method. The third part is the specific methods of data acquisition and processing, and the fourth part is the conclusion of this paper.

\section{The planning of spatiotemporal data acquisition and processing}

\subsection{Acquisition and processing specifications}

The digital acquisition specification of Ansai waist drum is introduced as follow due to the characteristics of motion capture system, which includes acquisition site, motion capture equipment, and post-processing software, as well as data storage format.

\subsubsection{Acquisition site}

There are two optical motion capture methods, the one is the active motion capture system and the other is the passive motion capture system. The active motion capture system capture the signal of the autonomous luminous source by a camera, then generate motion data,

"Corresponding author's e-mail: wangxupeng@xaut.edu.cn 
but it needs to be performed in a dark place and many data lines need to be placed on the capture object. Whereas, the passive motion capture system capture the reflection signal of light source from the marker, which is not affected by the environment. So in this paper, the latter is used.

\subsubsection{Motion capture equipment}

Generally, there are 8-12 cameras in the optical motion capture system, which can be expanded or deleted according to the actual situation. Cameras are placed in a circle or a square, as shown in Figure 1. The higher capture rate and resolution of the camera, the higher capture precision of motion track. In addition, the reflective markers of the light source should be arranged in important motion joints of the human, and the joint freedom also needs be considered, as shown in Figure 2.

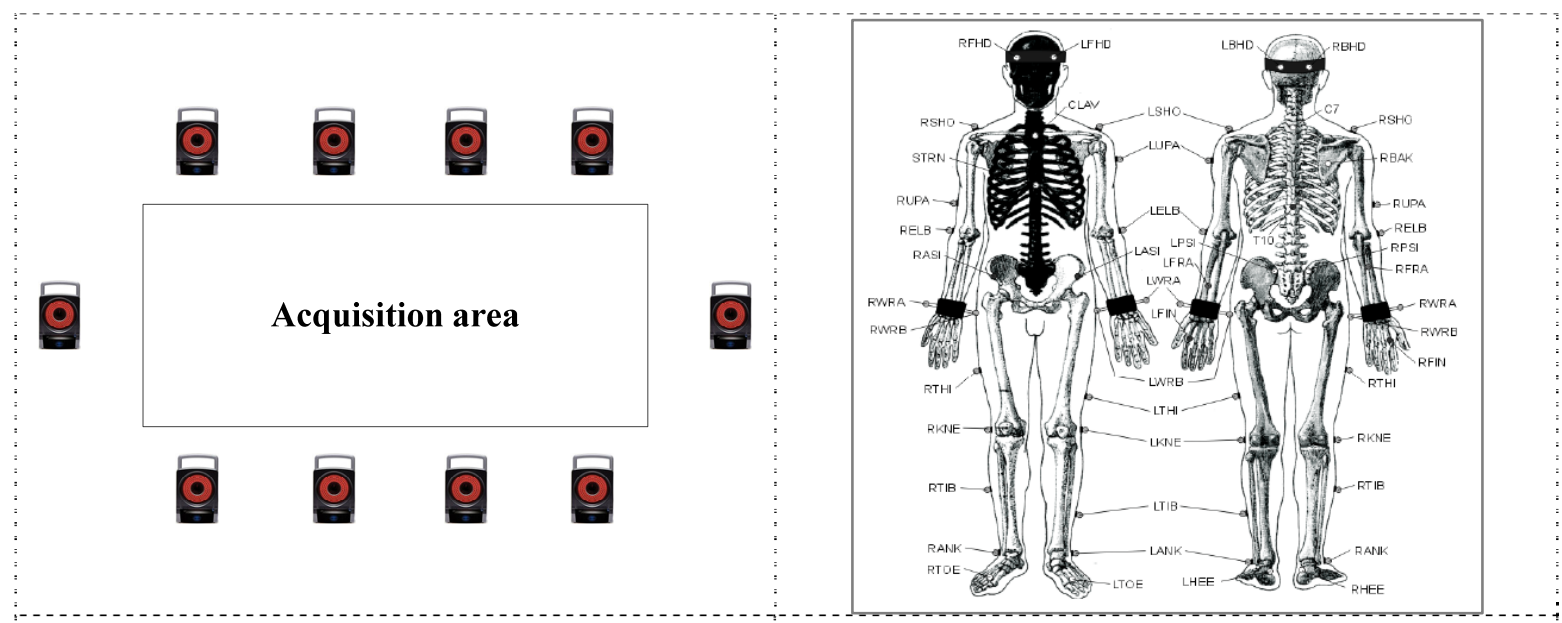

Figure 1. Camera layout of motion captures system.

Figure 2. Reflective markers of human body light source.

\subsubsection{Post-processing software}

The motion capture system can seamlessly interface with mainstream 3D software, such as Motion Builder, Maya, 3DMax et al. All post-processing software support many formats as follow, biped, CAT bone capture, C3d, FBX, BIP and others. At the same time, it can repair small test data breakpoints. In this paper, Motion Builder is used as the post-processing software.

\subsubsection{Data storage format}

For sharing of data, the captured data is processed into common formats, such as BVH, ASF \& AMC and c3d, and the $\mathrm{c} 3 \mathrm{~d}$ format is used in this paper.

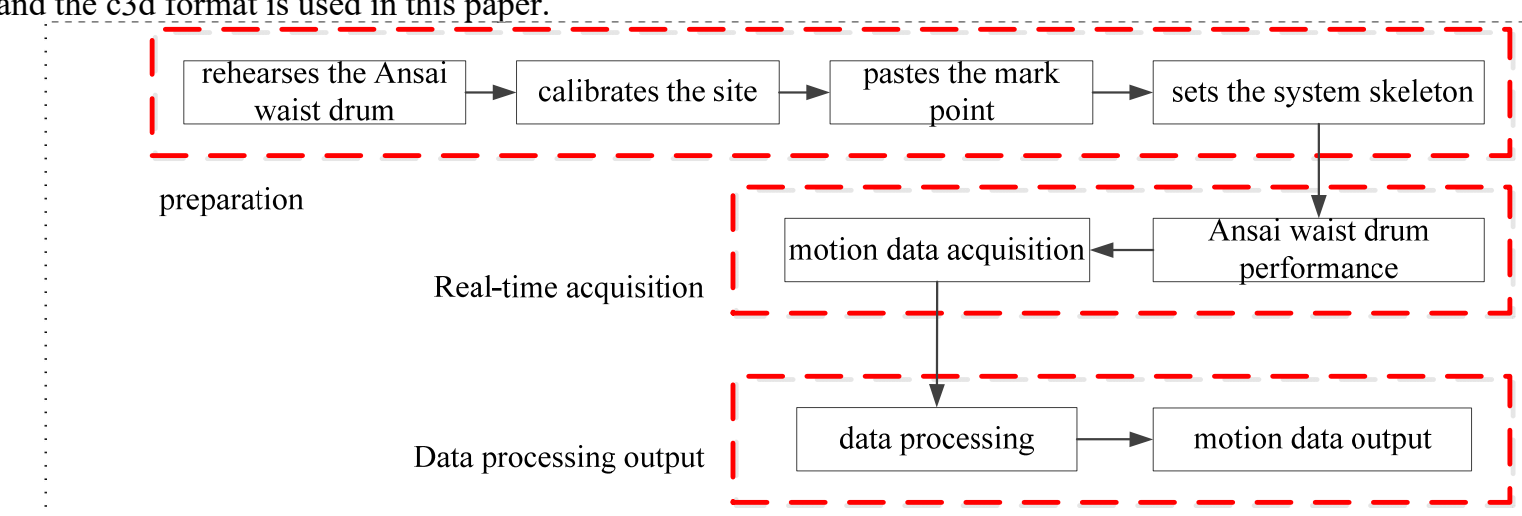

\subsection{Acquisition and processing flow}

The data acquisition and processing flow of Ansai waist drum is shown as Figure 3. It mainly includes the following steps, preparation(the inheritor rehearses the Ansai waist drum, calibrates the motion capture site, pastes the mark point on the inheritor's body, and sets the system skeleton), real-time acquisition(Ansai waist drum performance and motion data acquisition), and data processing output(data processing, motion data output). In the experiment, spatiotemporal data of all reflective markers can be collected, and each motion date is recorded as c3d format.

Figure 3. Flow chart of data acquisition and processing. 


\subsection{Laboratory equipment}

In this paper, Vicon motion capture system is used, which is produced and developed by the British company OXFORDMETRICSLIMITED, and is the first optical system designed for motion capture in the world. Vicon motion capture system was first used in the military field, and then gradually used in medical, sports, engineering, biology and other civil fields [9].

The Vicon motion capture system is consisted by follow equipments, which contain a set of networkconnected Vicon MX motion capture cameras, a Giganet device, and a PC host. These components establish a complete three-dimensional motion capture system. Table 1 shows a list of key components.

Table 1. Component of Vicon motion capture system [9].

\begin{tabular}{ll}
\hline Component Name & Description \\
\hline MX Camera & $\begin{array}{l}\text { Infrared camera, illuminator, lens, optical filter and } \\
\text { connecting wire, etc., recording the motion track }\end{array}$ \\
& $\begin{array}{l}\text { The distributed architecture consists of MX NET, } \\
\text { MX Link and MX Ultranet HD, which is used to } \\
\text { adapt to MX cameras and third-party hardware } \\
\text { devices in the system. }\end{array}$ \\
MX Component & $\begin{array}{l}\text { Install the Vicon nexus and Vicon polygon software } \\
\text { for data processing. }\end{array}$ \\
PC Host & $\begin{array}{l}\text { Vicon motion capture system and third-party test } \\
\text { system hardware equipment information }\end{array}$ \\
Vicon Data station ADC Patch Panel & Connect the components together \\
MX Dedicated Connecting wire & T-shaped correcting mount, for accurate calibration \\
of the Vicon MX system
\end{tabular}

It needs to be noted that the camera is equipped with a Vicon Vegas sensor, which can achieve high resolution, high capture frequency, and high precision, as well as long-distance capture. By capturing reflection markers on the body, a three-dimensional data motion acquisition and analysis system is set up. The three-dimensional coordinates of the reflection markers can be obtained by calculating the points scanned from different cameras at the same sampling time, and through kinematic analysis, the change laws of physical quantities such as displacement, velocity and acceleration of human body are obtained. During the capture process, even if the local capture mark points are blocked by the body, the satisfactory output data can still be obtained after processing by the software.

\section{Data acquisition and processing method}

\subsection{Data acquisition}

\subsubsection{Preparation}

Nine main motions of Ansai waist drum were selected to capture in experiment, which include XiaoChanYao, MaBuHengYi, and ChanYaoGuoDang et al. All motions should be repeated captured for three times. In addition, before the data acquisition the test participation needs to perform an action rehearsal. Then, the Vicon optical motion capture system was calibrated by a T-shaped correcting mount, in order to ensure the system can record the whole body reflective markers, as shown in Fig 4. And a total of 39 reflective markers are pasted on the whole body of the inheritor, which is follow the pasting position of the reflective markers of the human light source shown in Fig. 2, pasting of reflection markers as shown in Fig 5. 


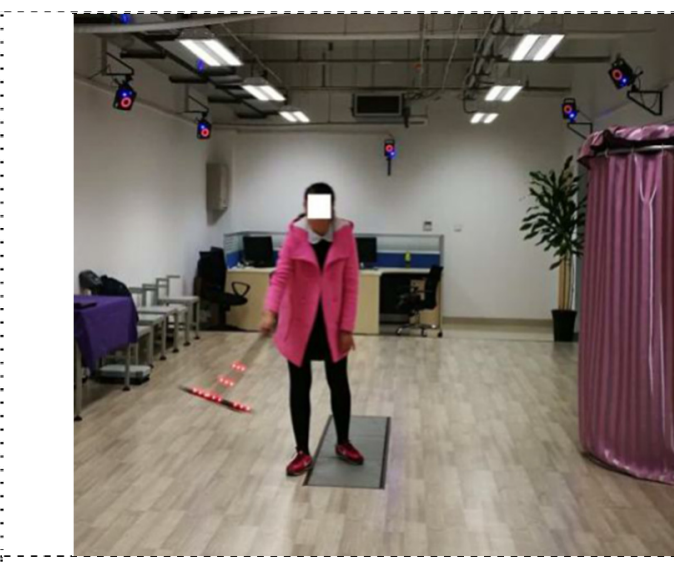

Figure 4. System calibration.

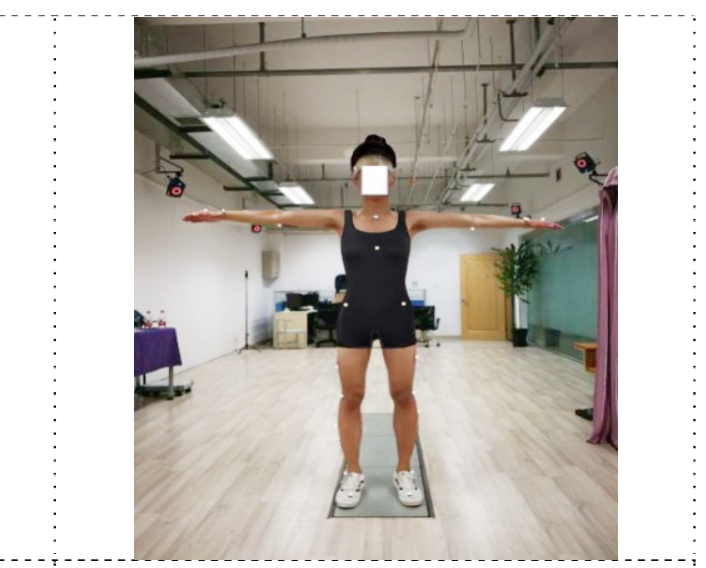

Figure 5. Pasting of reflection markers of body light source in the experiment.
The specific positions and definitions of the points are as follow, which contain Head (named RFHD, LFHD, LBHD, RBHD, respectively), Body torso (named C7, T10, CLAV, STRN and RBAK, respectively), Upper limb (named LSHO, RSHO, LUPA, RUPA, LELB, RELB, LFRA, RFRA, LWRA, RWRA, LWRB, RWRB, LFIN and RFIN, respectively), and Pelvis (named LASI, RASI, LPSI and RPSI, respectively), as well as Lower limb (LKNE, RKNE, LTHI, RTHI, LTIB, RTIB, LANK, RANK, LTOE, RTOE, LHEE and RHEE, respectively). Similarly, after the reflective markers are pasted, they need to be tested and calibrated.

\subsubsection{Real-time acquisition}

When the preparations were finished, the test participation stood in adjusted area for real-time acquisition. The acquisition motion motions were carried out successively, there were 9 groups of motions in total, and each group of motions was tested for 3 times. The Vicon motion capture system would automatically record the spatiotemporal coordinates of the inheritor's joint nodes. These coordinates would be analyzed and processed in the later stage with the help of Vicon Nexus software.

\subsubsection{Data output}

The data from the real-time acquisition were analysed using Vicon Nexus software. The three-dimensional coordinates of each marker could be used to automatically calculate the physical quantities, such as the change in the angle of each joint, and the torque of each joint, then finally outputted a total of 27 Excel table data in .csv format.

\subsection{Data processing}

\subsubsection{Excel data processing}

The spatiotemporal data of 39 markers were collected by Vicon motion capture system, which would be used as the standard motion database for Ansai waist drum beginners as self-study and learning evaluation. Therefore, the data processing should also be consistent with the data of acquisition equipment used by Selflearning users in the later period.

In this paper, Kinect V2 was selected as the acquisition device for self-learning users in the later period. The corresponding relationship between the markers of the Vicon motion capture system and the Kinect V2 Skeleton points are shown in Table 2, which contain a total of 20 marker points. For example, the Wrist_Right marker point in the Kinect V2 corresponds to the intermediate value of the vector, which is formed by the two points RWRA and RWRB in the output data of the Vicon system.

Table 2. Joints corresponding to vicon and Kinect V2 [9]

\begin{tabular}{llll}
\hline Kinect & Vicon & Kinect & Vicon \\
\hline Shoulder_Right & RSHO & Shoulder_Left & LSHO \\
Elbow_Right & RELB & Elbow_Left & LELB \\
Shoulder_Center & CLAV & Spine & STRN \\
Hip_Right & RASI & Hip_Left & LASI \\
Knee_Right & RKNE & Knee_Left & LKNE \\
Ankle_Right & RANK & Ankle_Left & LANK
\end{tabular}




\begin{tabular}{llll} 
Foot_Right & RTOE & Foot_Left & LTOE \\
Hand_Right & RFIN & Hand_Left & LFIN \\
Wrist_Right & RWRA, RWRB & Wrist_Left & LWRA, LWRB \\
Head & RFHD, LFHD, RBHD, LBHD & Spine_Base & RASI, LASI \\
\hline
\end{tabular}

In addition, the acquisition error influence on spatiotemporal data is inevitable. Therefore, the data obtained from the three acquisitions are fitted by finding the geometric mean value, and then used as the standard data for the operation, which can be calculated as

$$
Y_{j}=\sqrt[n]{\prod_{i=1}^{n} x_{j}}, n=20, \mathrm{j}=1,2,3
$$

where, $Y_{j}$ is a standard value of spatiotemporal data corresponding to a marker point in three experiments, $x_{j}$ is a test value of spatiotemporal data for a joint point during a single experiment.

\subsubsection{Skeleton data processing}

The standard motion spatiotemporal data are stored as .c3d format, which can be imported into Motion

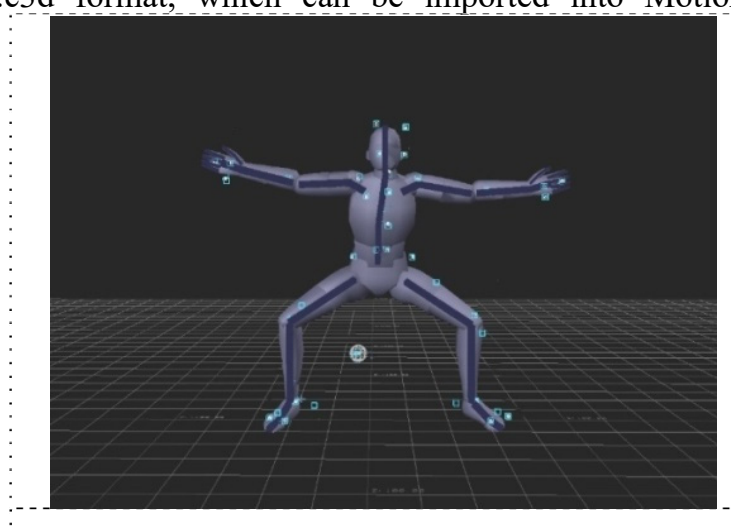

Figure 6. Skeleton data points.
Builder software to view and edit. Then changed into. fbx format data for creating skeletal animation, and visualized the skeleton data in unity3d. Finally, it can be used in the interactive learning of Ansai waist drum. Take the MaBuHengYi as an example. First, the spatiotemporal data with .c3d format are imported into Motion Builder software for preliminary verification. Second, the .c3d format skeleton data verified were accurately bound on the corresponding part of the actor, so that the actor could be driven by the standard data, as shown in Figure 6. Then, the actor was characterized and converted into. bvh format data with skeleton, as shown in Figure 7. Finally, the data with. fbx format were recognized by Unity3D software, and used to visualize the motion of Ansai waist drum.

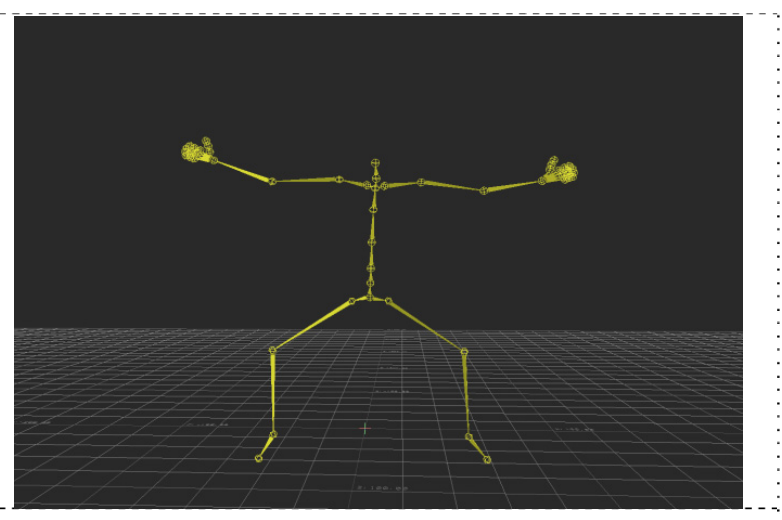

Figure 7. Bvh format of motion.

\section{Conclusion}

For the existing problem of inheritance and protection for Ansai waist drum, especially the inheritance mode is single, and mainly relies on the master-apprentice style and family style. By detailed description and examples, the paper introduces a digital method to acquire and process spatiotemporal data of Ansai waist drum inheritor, which is the basis and play a critical role in development of interactive studying system for beginners. It also needs to be noted that the method also can be a reference for inheritance and preservation to sports and dance intangible cultural heritage projects.

\section{Acknowledgments}

This work was supported by Research Foundation of the major theoretical and practical problems of Social Sciences in Shaanxi Province (Grant No. 2019Z096), and Foundation from Xi'an University of Technology (Grant No. 106-451619016). The authors would like to express their appreciation to the agencies.

\section{References}

1. Zhao, D.G. (2011) Research on the development prospect of Ansai waist drum from the perspective of intangible cultural heritage protection. Journal of Shaanxi University of science and technology, 29(03):183-186.

2. Wang, T.J, Fan L.H, Wang, H.L. (2016) Research on the development and inheritance of Shanxi Ansai waist drum. Zhejiang sports science, 38(03):54-55+92.

3. Gao, P.F. (2014) A study on the historical changes and inheritance of the waist drum movement in Northern of Shaanxi. Sports and Science, 35(03):78-82.

4. Marcello, C, Alessandra S, Rosario L. (2011) Virtually preserving the intangible heritage of artistic handicraft. Journal of Cultural Heritage. (12):82-87.

5. Jacky C.R, Howard, Jeff K.T. (2011) A Virtual Reality Dance Training System Using Motion 
Capture Technology. IEEE Transactions on learning technologies. 4 (2):187-195.

6. Shi, L. (2015) Digital protection of movement capture technology for dance of intangible cultural heritage. Yunnan Arts University.

7. Yu, T. (2013) Research on digital protection of Taijiquan based on action capture. Sun Yat-sen University.

8. Chen, J.Y. (2015) Research on the design of interactive Guangling paper cutting game. Harbin Institute of Technology.

9. Sun, C.M. (2013) Research on digital technology of folk dance intangible cultural heritage. Central China Normal University. 